\title{
Analysis of the Expansion of Space and a Theory of the Big Implosion
}

\author{
Paul Gradenwitz \\ Guizhou University, Guiyang, China \\ Email: paul.gradenwitz@bluewin.ch
}

How to cite this paper: Gradenwitz, P. (2018) Analysis of the Expansion of Space and a Theory of the Big Implosion. Journal of High Energy Physics, Gravitation and Cosmology, 4, 31-47. https://doi.org/10.4236/jhepgc.2018.41005

Received: November 2, 2017

Accepted: January 9, 2018

Published: January 12, 2018

Copyright $\odot 2018$ by author and Scientific Research Publishing Inc. This work is licensed under the Creative Commons Attribution International License (CC BY 4.0).

http://creativecommons.org/licenses/by/4.0/

\section{cc) (i) Open Access}

\begin{abstract}
In this article, the model of an expanding universe, which is more than a century old and evolved into the $\Lambda$-CDM, is found to violate causality. This is shown by constructing the lightcone, which is relevant for any point in space, and by calculating the curvature for that point. From these calculations it is concluded that for any homogeneous and isotropic energy density in space, the geodesic of a test mass in it is always straight. With that, it follows that space is flat for any energy density. Furthermore an alternative theory is proposed as a demonstration that, with different explanations of the astronomical observations, a theory can be constructed that isn't in contradiction with General Relativity.
\end{abstract}

\section{Keywords}

Cosmology, Gravitation

\section{Introduction}

In the last $100+$ years cosmology has made an enormous leap from a view of the cosmos, where the solar system is in the center, surrounded by stars and nebulae, to an exact science with detailed knowledge about a vast, seemingly, expanding universe filled with galaxies, gas and other unknown components. In the time when Einstein derived his theory of General Relativity (GR) [1].

$$
R_{\mu \nu}-\frac{1}{2} g_{\mu v} R+g_{\mu \nu} \Lambda=\frac{8 \pi G}{c^{4}} T_{\mu \nu}
$$

that proved to be better than Newton's theory of gravity [2], and Hubble mapped out space as it surrounds us and the galaxies in there [3], we did not yet have a clear view of cosmology. There was no knowledge of an origin, no knowledge of expansion, no knowledge of a background radiation and no idea that space could 
be filled with dark energy or dark matter. Around the same time also the FWRL metric (Friedmann, Walker, Robertson, Lemaître)

$$
\mathrm{d} s^{2}=-c^{2} \mathrm{~d} t^{2}+R_{0}^{2} a(t)^{2}\left(\mathrm{~d} r^{2}+r^{2}\left(\mathrm{~d} \theta^{2}+\sin ^{2} \theta \mathrm{d} \phi^{2}\right)\right)
$$

was introduced and Friedmann derived equations that describe how the scale factor of space is dependent on the energy density in it [4].

$$
\begin{gathered}
H^{2}=\left(\frac{\dot{a}}{a}\right)^{2}=\frac{8 \pi G}{3} \rho+\frac{2 E}{a^{2}} \\
\left(\frac{\ddot{a}}{a}\right)^{2}=-\frac{4}{3} \pi G \rho .
\end{gathered}
$$

Hubble discovered that all galaxies are receding from us with a speed proportional to their distance [3] He refined what Friedmann and Lemaitre, out of the analysis of the general relativity equations [5], already had predicted, that space might be expanding. This led to the result of a theory describing how space once was a dense point and since then expanded; the theory of the Big Bang. Einstein, who already had noticed that his GR didn't allow a stable universe when the energy density was not zero, and had inserted a constant $-\Lambda$-to his equations to allow for stability, removed this constant and called it his biggest error. Since then the theory of the Big Bang has seen victory over victory against other theories. The predictions made based on this theory, were repeatedly confirmed. The predicted background radiation was discovered, the abundance of the main elements was in accordance with it, the fact that the night sky is dark supported a universe of limited age, the extreme smoothness of the cosmic background radiation could be explained by an initial inflation and the discovery of the acceleration of the expansion brought Einstein's discarded $\Lambda$ back into the equations. This resulted in the current concordance model the $\Lambda$-CDM (a theory of a universe influenced by $\Lambda$ and cold dark matter). Surveys of COBE [6], WMAP [7], and PLANK [8] have refined the understanding of the total composition of the content of the universe in dark energy, dark matter and baryonic matter. Radiation is said to have had a role in the early universe but by now its influence has faded away. This very successful theory has also left us with some big questions.

- Until now there is no satisfactory answer on what is dark energy.

- There is still a big search ongoing to find any particle that could be responsible for dark matter.

- An attempt to explain dark energy as the energy density of the vacuum had devastating results as the expected value based on cosmological observation turned out to be $10^{120}$ times smaller than the value expected by quantum physics.

These three problems cast a shadow on the validity of the $\Lambda$-CDM.

The above-mentioned equations use 4-dimensional spacetime and make no difference to the fact that the three space dimensions $\mathrm{x}, \mathrm{y}$, and $\mathrm{z}$ are bi-directional and the one time dimension $t$ is unidirectional. As is stated for example in [9] on page 10: 
"Therefore the division of Minkowski space into space and time is a choice we make for our own purposes, not something intrinsic to the situation".

This is of importance to be able to derive the GR equations. The success, that the GR equations have, shows that it is a good thing not to make a distinction between space and time. To make no difference between the time dimension and the space dimensions in the derivation of the equations is something different to the interpretation of the meaning. Gauss's Law for gravity states:

The gravitational flux $\Phi$ through any closed surface is proportional to the enclosed mass.

With respect to the above-mentioned unidirectionality of the time dimension we have to question what effect this has on this law. Since, as is done in the above mentioned lecture notes [9] on page 109, the derivation of the right-hand side of the GR Equation (1) starts with Poisson's equation that is directly derived from Gauss's law, any consequence of the unidirectionality of the time dimension on this law has also an effect on the interpretation of the GR. The present article tries to come, via a more computational path, to solutions to the three above-mentioned problems by reanalyzing the validity of earliest conclusions of the theory and to propose another theory that might be able to answer some of them. The presentation of this newer theory isn't supported by detailed mathematical equations. As such it only gives a direction to search for new answers to old questions.

\section{Description of Principles}

\subsection{Principles List}

We begin with listing how in this article some basic principles are used.

- Spacetime-The Universe is described as 4-dimensional spacetime. 4-dimensional spacetime has three spacelike bi-directional dimensions and one timelike unidirectional dimension.

- Space-Space itself is, in this 4-dimensional spacetime, a 3-dimensional brane. Space only exists in the present. It no longer exists in the past and does not yet exist in the future. With this we also make a definition what is called present, past and future.

- Causal connection-The future of any point $\mathrm{P}$ in space can only be defined by the influence of any other point at a given time in the past as long as this other point is located inside a 3-dimensional cone in 4-dimensional spacetime with its tip in the present, its axis along the time direction through $\mathrm{P}$ and its radius at any time equal to the light speed multiplied by the time difference between the point at the given time and the present. Points outside the above-described cone have no relation to the point $\mathrm{P}$. They are nonexistent for P.

- Lightcone-The above-described cone is another 3-dimensional brane. It touches space in one point. It is best de-scribed in polar coordinates $\mathrm{r} \Theta \Phi$. 
Here $\mathrm{r}$ is a pure timelike dimension and both $\Theta$ and $\Phi$ are space like dimensions.

- Geodesic-A geodesic is the path in spacetime that a mass will follow if no external forces act upon this mass. It is a straight line if in its second derivative and in its higher derivatives there are no spacelike components in its equation.

- Worldline-The union of points along the time axis that follows a point in space is called a worldline. The length of the worldline is defined as the time since start of the universe times the speed of light. The time in the past is counted as negative from the present and the time to the future is counted positive. The worldline length of the present is the equal to the age of the universe, $T_{\text {universe }}$, times the speed of light, $c$. Where $T_{\text {universe }}$ is a positive value. A point in the past at time $t=T$ has a worldline length equal to $\left(T_{\text {universe }}+T\right) \times$ $c$.

Any point in space has no causal connection with any other point in space. It has a causal connection with a past version of another point but never with the present version. Only the present version of a point exists. Only the influence of a past version of other points defines the future of a present point.

Space is flat if, through any point of space, the geodesic is a straight line.

In discussions about GR it is usual to call a point in 4-dimensional spacetime an event. Here we use the normal word "point" because both space and a lightcone are 3-dimensional.

\subsection{Assumptions}

1) Space has a finite age;

2) Space is filled with a homogeneous isotropic energy density.

These assumptions have the consequence that any moving of particles is ignored. Moving of one particle would break homogeneity and would only be cancelled if other particles compensate this. Thus for space the end result would be the same as when no particle is moving.

\subsection{Question}

How will space react under the influence of the energy density in it? Will space have a curvature that depends on the amount of energy density in it?

\section{Derivation of an Answer}

To analyze the effect that any energy density has on space we can derive the geodesic through any point that a test mass would follow.

Space, the 3-dimensional brane in the present, can be described as a union of unrelated points. If space is filled homogeneously and isotropic with energy density, then the solution for any point is valid for all other points.

We refrain to use the GR equations here because, in order to come to these equations, Einstein had to make use of Gauss's theorem. This theorem was made 
in a time when there was no notion of the speed of light and a lightcone. This complicates the validity of this theorem and with that puts a question mark next to its use. Since the problem we have constructed is simple, we shall use a different method.

The derivation is made with an algorithmic method. This means that we don't try to derive a solution analytically, but that we try to compute the solution for every point. Similar to the beautiful simulation of the universe in PKDGRAV3 [10], where the light cone is calculated for each of the more than a trillion particles and a computation is made based on the intersection of the worldline of another particle with this light cone, here we too construct a lightcone for a point in the present and see how many other points interact with this point when their worldline intersects the cone. This method takes into account that time is unidirectional.

\subsection{Derivation}

We start with one arbitrary point $\mathrm{P}$ of space and define that as the center of our coordinate system. It is under the influence of the energy density within the cone as mentioned above. Since space has a finite age, according to Assumption (1), the height of the cone is the age of space and the radius at the base of the cone is the age of space multiplied by the speed of light. This cone is the total universe that is relevant for our chosen point. In this cone only the chosen point $\mathrm{P}$ is in the present. All other points are in the past.

The cone is a brane. That means that it is a 3-dimensional surface in 4-dimensional space-time. In the same way that a 2-dimensional cone in a 3-dimensional space has a surface, an inside, and an outside, this 3-dimensional cone also has a surface, an in-side, and an outside. As for a 2-dimensional cone its inside and outside is 3-dimensional, the inside and outside of the lightcone is 4-dimensional. Any point inside of the cone can have a causal connection with the present point at the tip of the cone. That connection is based on scattering. Any point outside of the cone, even if it is in the past, can't have a causal connection with the present point. Through scattering even a past version of the present point can have effect. But in the end, the final effect always is from another point at the surface of the cone. For these reasons we have to limit ourselves to the influence that a point, at the surface of the cone, has on the present point. The influence that any other point of the brane surface, in the past, has on the point in the present is the result of the lightcone for that other point. In that latter cone is the scattering we mentioned before. It consists only of points that are equal or inside the primary cone. Every point, whose influence has travelled, with the speed of light, over the same amount of time to reach the present point, is located in this brane on a spherical surface with a radius equal to the travel distance of that influence. That distance of that point on that surface neither needs to be the distance of the point in the past to the present point as it was when they had the same worldline length, nor to be the distance to that point as that is in the present 
where they both also have the same worldline length. Only under the condition that space is neither expanding nor contracting these distances might be the same.

To obtain the influence, which this cone has on our chosen point $\mathrm{P}$, normally we use Einstein's GR equations and, to describe the universe, we use the FWRL metric. When we fill in the metric and the energy density distribution in the GR then we will come to the conclusion that space is curved in this universe. This is what is shown in Equation (3) and Equation (4)

Because of Assumption (2) we can easily find the solution of the geodesic for our chosen point. Our described universe, that universe that has a causal relation with our chosen point, is a globe. It has a center in our chosen point and surface at the start of space. This globe does not need to have a homogeneous density. In the case that the scale of the universe changes in time the energy density of our lightcone, of our globe, can have a point symmetric energy density. In this case each point with the same distance to the center has the same energy density.

The geodesic of the center of this, for the point $P$ relevant, universe, filled with energy density as in Assumption (2), is the same as the geodesic of this whole, for the point $\mathrm{P}$ relevant, universe. Since it makes no sense to define the start of space to be moving in space like directions, we have to conclude that the geodesic of our chosen point can have only timelike components in its second derivative of its equations. Any other point in our described universe under normal derivations seems to have a geodesic with space like components in its second derivative of its equations but, next to the fact that it no more exists, this curvature is only true if the timelike dimension would be bidirectional. For space only the solution for the chosen point $\mathrm{P}$ is relevant.

To explain what is the difference between the normal derivations, which leads to the Friedmann equations, and our derivation, which takes into account that time is unidirectional, the following part is given.

A spherical shell is the volume between two concentric spheres with different radii $R_{1}$ and $R_{2}$. Let us state that $R_{1} \leq R_{2}$. The energy density within this shell volume between the two radii has no influence on the geodesic of any point inside a sphere with radius $R_{1}$.

From the view point of $\mathrm{P}$ any other point $\mathrm{P}^{\star}$, of our light cone, outside the chosen center point $\mathrm{P}$ has a spherical shell of energy density outside with inner radius $\mathrm{R}_{1}$, equal to the distance to the center point $\mathrm{P}$, and outer radius $R_{2}$, that is equal to the distance between the center point $\mathrm{P}$ and the start of the universe. This shell has no influence on the geodesic of the other point $\mathrm{P}^{\star}$. According to Gauss's law all energy density inside the sphere with radius $R_{1}$ has an influence. This is used to arrive to Equations (3) and (4). However, since our light cone has, in its radial direction, a pure timelike dimension, where, for point $\mathrm{P}^{\star}$, any point inside the sphere with radius $R_{1}$ is in the future, the total energy density in this sphere does not exist for the point $\mathrm{P}^{*}$. Gauss's law collapses because the surface of the sphere with radius $R_{1}$ encloses an amount of energy that is nonexistent for 
any point outside of this surface. The lightcone for any point $\mathrm{P}^{*}$ on that surface starts there and is only congruent with our initial lightcone for the extension of the line from our point $\mathrm{P}$ to point $\mathrm{P}^{\star}$ in a pure timelike direction from $\mathrm{P}^{\star}$ to the start of the universe. Any other point on the surface of that other lightcone for $\mathrm{P}^{*}$ is not part of the surface of our initial lightcone but older than $\mathrm{P}^{\star}$, where older means that its worldline is shorter than the worldline of $\mathrm{P}^{\star}$. Because these two light cones only are congruent over one line, also the outer shell, that is real for point $\mathrm{P}$, does not exist for point $\mathrm{P}^{\star}$. With this neither the inside nor the outside part of our light cone have any influence on the geodesic of $\mathrm{P}^{\star}$. Also the inside sphere will always give a curvature where the second derivative of the geodesic has only a component to the center of gravity of the sphere and with the Assumption (2) this is in the time direction towards the future. With that any influence would only give timelike components in the second derivative of the geodesic and not add to curvature.

If we want to get the geodesic for any other point $\mathrm{P}^{\star}$ in space-time we have to start all over again from that new point $\mathrm{P}^{\star}$. We have to define that other universe, the cone, the brane, which is relevant for this new point $\mathrm{P}^{\star}$. Then we again will come to the conclusion that, at the moment when our new point is in the present, its geodesic can have only timelike components in its second derivative. Since this is valid for any point in space-time it is especially valid for any point in space, the 3-dimensional brane of the present.

Because space only exists in the present and only reacts, and not acts, on the past and also has, for any point, a geodesic that can have only timelike components in its second derivative, space will neither expand nor contract under the influence of any homogeneous isotropic energy density inside it. Since, for our solution, we have no limit in the age of the universe, our solution is valid for an age that is arbitrarily old.

As we have just described, Gauss's law implodes. Since Gauss's law is at the core of the GR it appears that in this case the derivation of the curvature of space, using the FWRL metric and the GR that leads to the Friedmann equations, gives a result that is invalid.

\subsection{Answer to the Question}

Space will not react to any value of energy density inside it as long as it is homogenous and isotropic.

\subsection{Consequence}

In many documents that handle the subject of space expansion like [11] it is mentioned that for moving frames curvature is different to a frame at rest. The result would be that, for a particle following that moving frame, space would seem to be curved. But since we have started with an assumption that only allows moving particles that are compensated by counter moving particles to maintain homogeneity, this observed curvature has effect on the moving particles but not on 
space itself.

Any inhomogeneous distribution can be interpreted as the sum of a homogeneous part and an inhomogeneous part. We have shown here that there is no relation between the curvature of space and the homogeneous part. That means that the homogeneous part can be any value larger or equal to zero. The inhomogeneous part will give rise to local curvature. That curvature will decrease the mean radius of the curvature of space at locations, where the density will increase, and increase the mean radius of curvature at other locations, where the density will decrease.

The total sum has one part, which can be any value, without relation to the curvature of space and one part with a relation to local curvature. This means that the sum can't have a relation to the curvature of space.

As a consequence the $\Lambda$-CDM theory of the expanding universe, where there is a relation between the energy density in space and the expansion rate of space itself, appears to be incorrect.

\section{Argumentation to Come to an Alternative to the $\Lambda$-CDM}

Next to the $\Lambda$-CDM there are several other theories proposed that try to solve the above mentioned 3 fundamental questions. How the question, what theory could best describe the observations, could be settled is subject of [12] where is shown how these different theories allow different wave modes of gravity waves and how the measurement of these modes with the gravity wave detectors could settle who is closer to reality. All these theories use the same derivation method to come to a different kind of Friedmann equations that then would have different effect on the expansion rate of the universe. Because we have shown here that this method doesn't take into account that time is unidirectional and that this leads to a different conclusion when we take this fact into account, we don't need to make large differentiation between these other theories and the $\Lambda$-CDM. The conclusion of our derivation in no way states that the GR equations are wrong. We only state that, in the case of homogeneous and isotropic energy density in the universe, the correct conclusion is that this energy density has no relation to the expansion rate of the universe.

The $\Lambda$-CDM describes a cosmos where space expands and all energy density inside it dilutes as long as other forces allow that. So galaxies, galaxy clusters, move away from each other drifting on the expanding fabric of space. All matter inside this cosmos, on any point in space and time, has the same physical properties. Local differences in density result in local gravitational gradients, curvature, that makes that matter drift together through space. This movement through space, while assuming an expanding framework of space, is subject of the calculations in PKDGRAV3 [10]. Because this simulation is a calculation of the motion of points of matter through space it is valid for any reference frame. This simulation obtains its results from initial assumed quantum fluctuations in the early universe. Without these initial fluctuations there would be nothing to compute. When ob- 
jects in space would not move in a homogeneously filled universe then why would space itself do that?

The mathematical equations that describe the relation of the contents of space, to space itself, are here shown to be disputed. Not the observations, that were the basis to develop the $\Lambda$-CDM, but only the theory, which explains these observations, should be disputed. If the expansion rate of the universe has no relation with the energy density in it, then we can search for other solutions, based on the observed phenomena, which can be an alternative to the $\Lambda$-CDM.

When Hubble interpreted his observations he made a choice that was widely accepted in the science community. He chose to keep the assumption that size is a constant. Based on his choice of a constant unit of size he had to conclude that the size of the universe is not a constant.

In the subsequent part a new theory is proposed. This theory is highly speculative, as long as it is not tested. Here we only focus on the question if there is a principal mathematical support that would allow this theory to be correct. It is hoped that the proposed theory is accepted as a challenge to search for new properties of matter, which were outside the scope of questioning about their existence until now.

\subsection{The Big Implosion Theory}

A possible alternative is a theory where we chose to abandon the principle that size is a constant. Instead of a constant local size we chose that the universe is stable in size so that the size of matter shrinks. Such a universe would be mathematically reciprocal to the $\Lambda$-CDM. The reference frame of this universe is often used. It is called a comoving reference frame (see Appendix A). That the change of reference frames makes no changes to the observations is also demonstrated in [13] and [14]. There a cosmology model is proposed where a scalar field influences the mass of everything. However, the expansion or contraction of space is still dependent on the energy density, the mass, inside of it. With this that theory ends up with the same problem as the $\Lambda$-CDM. Based on our conclusions it seems to violate causality. Where, in the $\Lambda$-CDM, the observed longer time duration and wavelength, of processes in the past, is explained because their information expanded with the expansion of the universe, in our proposed alternative theory this difference is explained such that mass, time, charge and size shrink proportional to each other. Also in this alternative theory matter, in any position in space-time, has a fixed mass, size, charge and time relation. But, contrary to the $\Lambda$-CDM, the absolute size can be different from matter elsewhere in the universe. Matter in earlier time has larger dimensions than matter in later time. With such universe the observed redshift is explained by shrinking of matter.

\subsection{Deductions from Observational Results}

The recent results from the LIGO [15] experiment (GW150914) were interpreted as that a $29 M_{\odot}$ black hole and a $36 M_{\odot}$ black hole merged into a $62 M_{\odot}$ 
black hole. In this event $3 M_{\odot}$ were converted into energy that was radiated out in gravity waves. As was proven before with neutron double stars momentum is converted into gravity waves by moving masses. Since the GR is valid over all scales every moving mass has to emit gravity waves.

Based on above considerations we have to conclude that all matter has to emit gravity waves and thus loses momentum. All matter has to shrink. Atoms have to shrink. We also have to consider that a system, that emits gravity waves in a certain frequency range, also can absorb gravity waves, in that frequency range. The transfer of momentum is a method used in space travel. The probe to Pluto was not possible without a gravity assist from Jupiter. Thus, atoms that are close to other atoms will emit AND absorb gravity waves. They will shrink less than atoms that are far away from other atoms. Objects, like planets, will shrink faster at their surface than in their core. Because, when matter shrinks, their mass shrinks linear with their size while their volume shrinks with the cube of their size, matter gets denser when it shrinks. Objects with a fluid content will see matter near their surface move down. Convection will happen. We can therefore call this process gravitational cooling. Normal thermal cooling will also generate convection in fluids. Both effects are on a different scale but have similarities.

The observation of gas, that emits its light far in the blue direction of the spectrum, explained as extreme hot intergalactic gas, can now be explained differently. That gas needs no heat source. It is gas that shrinks with maximum speed because of its large atom separation.

We have the observation that earlier matter is seen with larger wavelength of light. Here we have chosen to interpret this, as that this earlier matter was bigger. Based on this observation with this interpretation we deducted a possible explanation for why matter could shrink.

\subsection{How Does the Shrinking of Mass, Size, Time and Other Units Correlate with Each Other?}

We observe that in older time the wavelength of light is longer and that processes take longer to evolve. With an expanding universe this observation is explained with the expansion. While we here assume that the universe is constant in size we have to explain this with the change of the progress of time and the change of the size of matter. If we introduce time, as experienced by matter, with mattertime $T_{M}$ and the size of matter $S_{M}$ and that both are a function of the redshift parameter $z$ then we can describe our observation with:

$$
\begin{gathered}
T_{M}(z)=(1+z) T_{M}(0) \\
S_{M}(z)=(1+z) S_{M}(0)
\end{gathered}
$$

A reasoning how mattertime has to be slower if mass is larger is given here.

All time processes depend on the orbital configuration of matter. The second is the duration of $9,192,631,770$ periods of the radiation corresponding to the transition between the two hyperfine levels of the ground state of the cesium 133 atom 
at $0 \mathrm{~K}$. This SI definition refers to a physical process where we assume that the orbital parameters of the atom and its electrons are constant. If we want to explain the observations then we have to have a system where the time and the emitted wavelength scale equal. The orbital time is also the reference for the length, the meter. Our SI definition of a meter is: Length of the path travelled by light in a vacuum in $1 / 299,792,458$ seconds.

If we keep the speed of motion as a constant then we see that with double the radius of an orbit the orbital time is also double. In order to have a stable orbit at this double size we need also double the mass. We can derive that by analyzing the following equation where a small mass $m_{2}$ orbits a large mass $M_{1}$ as a function of the distance to the observer with the parameter $z$.

$$
F(z)=G \frac{M_{1}(z) \times m_{2}(z)}{r(z)^{2}}=\frac{m_{2}(z) \times v^{2}}{r(z)} .
$$

Equal to the redshift we can state that the size scales with $z$ as:

$$
r(z)=(1+z) r(0)
$$

Now we have to ask what is the relation between a mass at distance $z$ to a mass at distance 0 , here at the observer. We set that as:

$$
m(z)=\alpha m(0)
$$

Then we can rewrite Equation (7) as:

$$
F(z)=G \frac{\alpha M_{1}(0) \times \alpha m_{2}(0)}{(1-z)^{2} r(0)^{2}}=\frac{\alpha m_{2}(0) \times v^{2}}{(1-z) r(0)}
$$

We can rewrite Equations (7) and (10) so that the constant $G$ and the velocity $v$ are at one side and the rest at the other side. We chose for the Equation (7) the parameter $z=0$. Then we can combine them and simplify:

$$
\frac{\alpha M_{1}(0)}{(1+z) r(0)}=\frac{v^{2}}{G}=\frac{M_{1}(0)}{r(0)}
$$

From this it follows that:

$$
\alpha=(1+z)
$$

and with that we can conclude that also:

$$
m(z)=(1+z) m(0)
$$

While size and time scale linear, the speed (of light) stays constant. Larger atoms have larger orbits of their electrons. With the speed of an electron constant the orbital time is linear to its radius. An object in orbit around another object will remain in a similar but scaled orbit when the mass of the two objects scale linear to the orbit size. This model, where mass, time and size shrink linear to each other, is consistent with all observations made in astronomical history as is for all models that are based on coordinate transformation. We have constructed a mass, size and time relationship that explains the astronomical observations with a constant size universe. 
Larger matter that shrinks over time explains Hubble's observation of redshift, for further away and older galaxies. The interpretation of this redshift as motion away from the observer is an assumption that is valid under the assumption that the size of matter is constant. GR does not support moving matter along curved geodesics with conservation of momentum. The interpretation of this redshift, as the evidence that matter in the past was bigger, has the support of the GR. The shrinking of matter doesn't need to be linear. Accelerated shrinking is more natural than linear shrinking. In nature processes with exponential behavior are well known. A universe, where matter shrinking is accelerated, is in accordance with observations.

There is yet another constant in our physics system. That is the constant of charge of an electron. The orbit of an electron around a proton is not governed by gravity but by electrical charge. The same reasoning that leads to the conclusion that matter has to scale linear with the size is valid for the electrical charge.

Could we have observed these electrons of much larger size and charge? A particle has its own time. There is a lot of evidence that matter is ejected at relativistic speeds from supernova explosions and in jets of mass from neutron stars and black holes. The time for such matter is running much slower than the time for the environment. When such particles hit our cosmic particle detectors the only parameter we are able to detect is the amount of energy it gives off. There seems no clear upper limit on the detected energy of such events.

\subsection{Can the Universe Be Filled with Homogeneous and Isotropic Energy Density?}

Based on the calculations, that predict that all matter shrinks, and the deduction that space doesn't need to change its scale, we have to conclude that a model, where only the universe expands, can't completely describe the observations. Instead we come here with a model that describes all observed phenomena with a static universe and shrinking matter. As seen above, the energy density of space can be anything. The vacuum energy density as predicted by quantum physics of $10^{112} \mathrm{erg} \mathrm{cm}^{-3}$ can be adopted to be correct. All mass in the universe is in the order of $10^{-9} \mathrm{erg} \mathrm{cm}^{-3}$ and that is then a diminishing small fraction of the total energy density. With that a universe with a homogeneous and isotropic energy density could be close to the right model. This universe can be infinitely old (arbitrarily old), and with that there is no need for an explanation of its start.

\subsection{Predictions Made Based on the Shrinking Mass Theory}

A pure change to the comoving reference frame as the explanation of reality does change nothing to the observations. Since it is a mathematical transformation, observations out of the old theory can't disprove the new theory For example the decay of the orbital momentum in neutron double stars is measured and compared with predictions of the GR. The statement, which states that any deviation of this process, due to the shrinking of matter, would be large enough to be visible in the measurements, is not correct. The measurement, as seen from the com- 
oving reference frame, is done using measurement tools that scale the same way as the observed orbital decay. Thus the result has no deviation.

With this result we can see that a homogeneous shrinking matter universe can't be distinguished from an expanding universe. However in the expanding universe there is only one entity that is active in the change of size and that is the universe itself. In the shrinking matter universe each item of matter has to shrink based on assumed uniform laws of nature. That the laws of nature are uniform the same everywhere in space is a well-accepted assumption. These laws will show their effect on every atom according to the environment it is in.

Since the environment is not uniform the result has to be not uniform. This leads to a prediction that can be tested.

Mass, separated enough from other mass, will shrink with a different rate than mass embedded in a large amount of mass.

The proof of this prediction would be to send a space probe equipped with an accurate clock to a large distance from the earth and to observe that the speed of this clock is faster than a clock kept on the surface of the earth or in a place as deeply embedded in the earth as possible. This difference would be more than the GR would predict because of different gravity effects.

If we consider that then also the neutron double star orbital decay should show up then we have to remember that the radius of such star is extremely small. With such small radius the shrinking of mass happens everywhere close to the surface. It will need a much deeper understanding of the shrinking of mass to predict how much the deviation is of shrinking of mass on the surface of the earth compared to the surface of the neutron star (see Appendix B).

\subsection{Dark Matter}

Galileo initiated a development where the scientific theories of the Universe abandoned the concept that there is a fixed reference of place. Where before his time the main opinion was that the earth is at the center of the universe, now the opinion is that there is no center at all. With the analysis made here about the expansion of space, the leading theory about the universe seems to be based on a failing assumption that the size of the universe depends on the energy density in it. It assumes that there is an absolute reference of size. Here the science community is asked to test a new theory, which puts away with both an absolute reference of location and an absolute reference of size. With releasing these constants of size and location we could go one step further. Our observations show us that the movement of stars within a galaxy and the movement of stars within a cluster has a too high speed to remain gravitationally bound if only the visible matter would generate gravity. In terms of Newtonian equations we see that

$$
G \frac{M_{1} \times m_{2}}{r^{2}} \neq \frac{m_{2} \times v^{2}}{r}
$$

because the speed $v$ is too high. We can bring it back to equality by changing the value for $M_{1}$ or we can decide that our gravitational parameter $G$ is not constant. 
If each unit of matter is a separated entity of space, then its surface is a node, a zero, for the energy waves of the vacuum. Between two particles, masses, the energy density has to be smaller than outside the two because it is restricted to those frequencies that fit between the surfaces of matter. As a result the vacuum energy will push both together. With the extreme high value that is predicted by quantum physics we can imagine that the vacuum energy is acting here. The dark energy is seen as the force to push space apart in the $\Lambda$-CDM. Here the same reasoning is used to let vacuum energy in a larger void sweep an area of smaller voids, with lower vacuum energy, together. From this consideration follows, that, the energy density of the vacuum, has to be a function of the size of the volume of empty space. The gravitational parameter $\mathrm{G}$ would then be also a function of this average size of emptiness between matter. What we have mapped out, and interpreted, as concentrations of the elusive dark matter, with the same right could be interpreted as the variation of the gravitational constant G. The consideration is done using Newton's equation. This is sufficient to understand the issue. However we need the full GR equations if we would like to get quantitive solutions.

This modification of Newton's equations is known under MOND (Modified Newtonian Dynamics). A main explanation of the MOND assumes that the parameter $\mathrm{G}$ is a function of the acceleration itself [16]. With a proper choice of parameters it is possible to make this fit with normal galaxies. The largest acceleration forces are where the largest mass concentration is. With this explanation of the change of $\mathrm{G}$ it would give however a prediction, of the distribution for a system like the Bullet Cluster, that differs from the observation [17]. In the MOND theory the prediction is, that, with the bullet cluster system, the largest concentration of mass would be where the gas resides. However the observations show that the higher amount of invisible mass seems to be where the galaxies are. The gas clouds interact with each other. So in a collision their speed is changed. The stars and other objects move through each other mostly without collision. As a result the gas is trailing the stars. If dark matter does not interact with itself and stars then this also would move together with the stars. That would make the most mass, the dark matter, move with the stars. The observation favors the explanation of dark matter over the explanation of MOND that sees the highest concentration in the gas clouds.

In the theory, which is proposed here, the average size of empty space from one atom to the next is taken as a measure of how high the energy density of the vacuum is. Because in normal galaxies the densest gas follows the visible structure of the galaxy we see that the larger $G$ envelops the galaxies. In the bullet galaxy system the gas is dislocated from the galaxy centers. This makes these areas more devoid and thus we would expect here also with the stars the larger G. This is in agreement with observations.

\section{Conclusion}

Detailed analyses of the equations that form the basis of the $\Lambda$-CDM have re- 
vealed that they seem to contradict causality. Based on the fact that time is unidirectional, conclusions out of mathematical derivations, that do not take this fact into account, are questioned for their validity. An alternative theory is proposed that is shown to have the potential of answering some of the main unknowns of the $\Lambda$-CDM. With that, the big question on what is dark energy can be solved. There is no expansion and no accelerated expansion. There is accelerated shrinking of matter. Also another problem can be settled. Our discrepancy between theory and measurement of $10^{120}$ can be removed. With no restriction on the energy density content of the universe the measurement seems invalid and the larger number can be taken as correct. As a third, the dark matter could also be interpreted as the variation of gravitational parameter $\mathrm{G}$ over space.

\section{Acknowledgements}

The author would like to thank those people that encouraged and helped him to complete this paper.

\section{References}

[1] Albert, E. (1916) Die Grundlage der allgemeinen Relativitätstheorie. Annalen der Physik, 49, 769-822

[2] Newton, I. (1687) Philosophiae Naturalis Principia Mathematica. Guil \& Joh. Innys, London. http://www.wilbourhall.org/pdfs/newton/NewtonPrincipia.pdf https://doi.org/10.5479/sil.52126.39088015628399

[3] Hubble, E. (1929) A Relation between Distance and Radial Velocity among Extra-Galactic Nebulae. Proceedings of the National Academy of Sciences, 15, 168-173. https://doi.org/10.1073/pnas.15.3.168

[4] Friedman, A. (1922) Über die Krümmung des Raumes. Zeitschrift für Physik, 10, 377-386. https://doi.org/10.1007/BF01332580

[5] Lemaitre, G. (1927) Un univers homogène de masse constante et de rayon croissant rendant compte de la vitesse radiale des nébuleuses extra-galactiques. Annales de la Société Scientifique de Bruxelles A, 47, 49-59.

[6] Mather, J.C. and Smoot, G.F. (1989/1992) COBE Mission Design, Spacecraft and Orbit. Goddard Space Flight Centre, Greenbelt.

[7] Spergel, D.N., Verde, L., Peiris, H.V., Komatsu, E., Nolta, M.R., Bennett, C.L., Halpern, M., Hinshaw, G., Jarosik, N., Kogut, A., Limon, M., Meyer, S.S., Page, L., Tucker, G.S., Weiland, J.L., Wollack, E. and Wright, E.L. (2003) First Year Wilkinson Microwave Anisotropy Probe (WMAP) Observations: Determination of Cosmological Parameters. The Astrophysical Journal Supplement Series, 148, 175-194. https://doi.org/10.1086/377226

[8] European Space Agency (2015) Planck Publications: Planck 2015 Results. http://www.cosmos.esa.int/web/planck/publications

[9] Carroll, S.M. (1997) Lecture Notes on General Relativity. University of California, Santa Barbara.

[10] Potter, D., Stadel, J. and Teyssierl, R. (2017) PKDGRAV3: Beyond Trillion Particle Cosmological Simulations for the Next Era of Galaxy Surveys. Computational Astrophysics and Cosmology, 4, 2. https://doi.org/10.1186/s40668-017-0021-1

[11] Baez, J.C. and Bunn, E.F. (2006) The Meaning of Einstein's Equation. 
http://math.ucr.edu/home/baez/einstein/einstein.pdf

[12] Corda, C. (2009) Interferometric Detection of Gravitational Waves: The Definitive Test for General Relativity. International Journal of Modern Physics D, 18, 2275. https://doi.org/10.1142/S0218271809015904

[13] Wetterich, C. (2013) Universe without Expansion. Physics of the Dark Universe, 2, 184-187. https://doi.org/10.1016/j.dark.2013.10.002

[14] Wetterich, C. (2014) Hot Big Bang or Slow Freeze? Physics Letters B, 736, 506-514. https://doi.org/10.1016/j.physletb.2014.08.013

[15] Abbott, B.P., et al. (LIGO Scientific Collaboration and Virgo Collaboration) (2016) Properties of the Binary Black Hole Merger GW150914. Physical Review Letters, 116, Article ID: 241102. https://doi.org/10.1103/PhysRevLett.116.241102

[16] Milgrom, M. (1983) A Modification of the Newtonian Dynamics as a Possible Alternative to the Hidden Mass Hypothesis. Astrophysical Journal, 270, 365-370. https://doi.org/10.1086/161130

[17] Clowe, D., Brada, M., Gonzalez, A.H., Markevitch, M., Randall, S.W., Jones, C. and Zaritsky, D. (2006) A Direct Empirical Proof of the Existence of Dark Matter. The Astrophysical Journal Letters, 648, L109-L113. https://doi.org/10.1086/508162 


\section{Appendix A: Reference Frames}

In General Relativity it is common to talk about many reference frames. General Relativity equations are such that they are valid in all kind of reference frames. In Astronomy we have also many reference frames. One common frame is our solar system. This frame is very useful to orient our self in space travel as far as we can go. However it is a reference frame with limited use. Another is a frame that is referenced to the surface of the earth. In these frames the universe is rotating. In them we find objects that move and obey special relativity, where nothing can move faster than the speed of light, but also many objects that don't obey special relativity. Most of the universe and space itself, in those reference frames, are zipping around in speeds that exceed the speed of light. The normal conclusion is that these chosen reference frames can't be the overall valid reference frame of space. The reference frame where space is expanding sees the distance of far away objects increase with a speed larger than the speed of light. That should be an indication that this reference frame also can't be the general valid reference frame. Because everything is energy $(E=m c 2)$, and because energy tends to flow, a reference frame where the size of matter is constant and part of the objects with respect to the frame seem to defy special relativity, is as much an arbitrary frame as the above mentioned solar or terrestrial reference frames. The only reference frame that has any movement of objects inside it obey the special relativity is the comoving reference frame. This makes the comoving reference frame a natural candidate for a general valid reference frame. In the here proposed theory this comoving reference frame is chosen. With that the universe is constant in size and matter properties shrink.

\section{Appendix B: Some Extra Thoughts}

A black hole has no internal moving objects. It doesn't emit gravity waves. Black holes that emerged in the early time before the re-ionization maybe never reduced in size. They could appear as super massive black holes in later time. 